\title{
Proximal Deletion 12q with a New Insight to Growth Retardation
}

\author{
Maria Sobol $^{\mathrm{a}}$ Ann-Charlotte Thuresson ${ }^{\mathrm{a}}$ Nathalie Palmberg ${ }^{\mathrm{b}}$ \\ Cecilia Soussi Zander ${ }^{a}$ \\ aDepartment of Immunology, Genetics and Pathology, Science for Life Laboratory, Uppsala University, Uppsala, \\ Sweden; ${ }^{b}$ Department of Neurology, Sachsska Children's Hospital, Stockholm South General Hospital, Stockholm, \\ Sweden
}

\section{Established Facts}

- Proximal deletions of the long arm of chromosome 12 have been described in about 20 patients with growth and developmental delay. No clear candidate gene has been identified yet.

- The ARID2 gene has been associated with intellectual disability and a Coffin-Siris-like phenotype.

\section{Novel Insights}

- The present case contributes to the common phenotypic features for patients with proximal deletion 12q such as developmental delay, growth retardation, broad forehead, large low-set ears, broad nasal bridge and/or nose, long philtrum, and widely spaced nipples.

- ARID2 is the only gene in common for patients described with a deletion at 12q12q13.1 and growth retardation $(<-2 \mathrm{SD})$.

- ARID2 single nucleotide variants do not correlate with severe growth retardation.

\section{Keywords}

ARID2 - Deletion 12q12q13.11 - Developmental delay · Growth retardation - SNP array

\begin{abstract}
Proximal deletion of the long arm of chromosome 12 is a rare chromosomal abnormality described in about 20 patients. Known deletions span the region from $12 q 11$ to $12 q 13$ and include the genes YAF2, AMIGO2, and NELL2. These are suggested as candidate genes for the key phenotypic features such as growth and psychomotor retardation. Here, we present a case with a 3.1-Mb interstitial deletion at 12q12q13.11.
\end{abstract}

The clinical observations of our patient overlap with the major common findings for published cases. The deletion detected in our patient does not involve the previously suggested candidate genes YAF2 and AMIGO2. We draw a correlation between proximal deletion $12 \mathrm{q}$ and ARID2 deficiency by comparing patients carrying gross deletions with a cohort of patients carrying small intragenic ARID2 deletions as well as patients with single nucleotide variants (SNVs) in ARID2. Growth retardation <-2 SD is present in cohorts with both gross and small deletions spanning ARID2. However, ARID2 SNVs do not correlate with severe growth retardation.

(c) 2020 The Author(s)

Published by S. Karger AG, Basel

\begin{tabular}{ll}
\hline KARGER & $\begin{array}{l}\text { (c) } 2020 \text { The Author(s) } \\
\text { Published by S. Karger AG, Basel }\end{array}$ \\
karger@karger.com & \\
www.karger.com/msy & $\begin{array}{l}\text { This article is licensed under the Creative Commons Attribution- } \\
\text { NonCommercial-NoDerivatives 4.0 International License (CC BY- } \\
\text { NC-ND) (http://www.karger.com/Services/OpenAccessLicense). } \\
\text { Usage and distribution for commercial purposes as well as any dis- } \\
\text { tribution of modified material requires written permission. }\end{array}$
\end{tabular}

Maria Sobol

Department of Immunology, Genetics and Pathology, Science for Life Laboratory Uppsala University, Rudbeck Laboratory C11

SE-75185 Uppsala (Sweden)

maria.sobol@igp.uu.se 
Chromosome $12 \mathrm{q}$ deletions are a heterogeneous group of genetic conditions that can be clustered into 3 major subgroups: (i) proximal deletions with breakpoints in 12q11q13; (ii) intermediate deletions with breakpoints in $12 \mathrm{q} 15 \mathrm{q} 21$, and (iii) distal deletions with breakpoints in 12q22q24. Moreover, a defined 12q14 microdeletion syndrome has been described in the literature [Lynch et al., 2011]. A variable degree of developmental delay as well as intellectual impairment was noted for most of the published cases. This fact is in agreement with the general observation that the majority of chromosomal rearrangements are associated with developmental and intellectual retardations as well as a broad spectrum of dysmorphisms.

Proximal deletions of the long arm of chromosome 12 have been described in about 20 patients. Five of these share a well-defined phenotype characterised by developmental delay with cognitive impairment, growth retardation, and decreased head circumference as well as a broad forehead, large low-set ears, broad nasal bridge and/or nose, long philtrum, and widely spaced nipples [Tonoki et al., 1998; Miyake et al., 2004; Failla et al., 2008; Carlsen et al., 2015; Weng et al., 2018]. All these patients have an overlapping deleted region at $12 \mathrm{q} 12$, where $Y A F 2$, AMIGO2 and NELL2 were suggested as candidate genes for growth and psychomotor retardation. However, no strong correlation or experimental proof have been shown for these genes to be associated with the mentioned phenotypic abnormalities.

In this study, we report a patient with a $3.1-\mathrm{Mb}$ deletion at $12 \mathrm{q} 12 \mathrm{q} 13.11$ contributing to the cohort of patients with proximal deletions at 12q. The present deletion does not span previously suggested candidate genes YAF2 and AMIGO2. Instead it delineates a minimal critical region for moderate to severe growth retardation with ARID2 as a candidate gene. We also compare this cohort with patients carrying ARID2 gene disruption and for the first time propose ARID2 as a candidate gene for moderate to severe growth retardation $(<-2 \mathrm{SD})$. We notice that ARID2 single nucleotide variants (SNVs) do not correlate with severe growth retardation, which may indicate diverse effects of different types of genetic variants on ARID2 protein function.

\section{Case Presentation}

The male patient was born at term after an unremarkable pregnancy and delivery; Apgar scores were 9-10-10. Birth weight was $3,210 \mathrm{~g}$ (15th centile/-1 SD) and length $47 \mathrm{~cm}(<10$ th centile/ $-2 \mathrm{SD}$ ). He had difficulties with breastfeeding and did not take on weight as fast as expected. The boy came to medical attention because of failure to thrive. Although his target height is around $1.80 \mathrm{~m}( \pm 0 \mathrm{SD})$, he has been growing on $-3 \mathrm{SD}$ for length, a bit below -2 SD for weight, and has a head circumference of -1 SD (Fig. 1A). Early on, he showed signs of developmental delay, especially in terms of oral motor function and expressive speech development. At 6 years of age, he is drooling and is still eating mashed food. He started walking unsupported at 18 months and still has some difficulties with fine motor activities. According to the parents, he was more like a 2-year-old at 3 years of age. He was toilet trained at 3.5 years of age and is wearing diapers at night at the age of 6.5 years.

Because of a slight strabismus, he is followed up by an ophthalmologist. Hearing is reported to be normal. Psychological assessment performed at age 6 concluded an expressive language delay, difficulties with transitions and changes of routines. Overall, it was concluded that he showed an impaired ability to social reciprocity and communication that was assessed to give a clinically significant functional impairment. However, there was no clear sign that he exhibited any stereotypical, limited or repetitive interests, and therefore he fulfilled the diagnostic criteria for atypical autism according to ICD- 10 .

At examination, one notices a short boy with a scaphocephalic head shape, long face, prominent forehead, slight strabismus, broad nose and nasal bridge, long philtrum, thin upper lip, higharched palate narrowed ventrally, widely spaced nipples, broadbased fingers, and hypoplastic toenails (Fig. 1B-F). He has a decreased muscular tone. For a detailed clinical description see Table 1 .

\section{Method and Results}

In view of the growth retardation, a hormone screening was performed at age 3.4 years. Thyroid function test was within the normal range (TSH $1.77(0.7-6.0) \mathrm{mlE} / \mathrm{L}$, FT4 $16.4(12.3-23)$ $\mathrm{pmol} / \mathrm{L}$, FT3 $5.7(3.7-8.5) \mathrm{pmol} / \mathrm{L})$, as well as the insulin growth factor 1 (IGF-1, $38(27-172) \mathrm{ug} / \mathrm{L}$, and $58 \mathrm{ug} / \mathrm{L}$ at age 3.5 years).

The patient was referred for genetic testing because of developmental delay and dysmorphism. Chromosomal microarray analysis was performed using CytoScan HD (Thermo Fisher) following the manufacturer's instructions. The analysis identified a 3.1-Mb interstitial deletion in the long arm of chromosome 12, arr[GRCh37] 12q12q13.11(43889138_47011108)x1. FISH analysis was performed on metaphase spreads from cultured blood lymphocytes using BAC probe RCPI-11 95K16 (Empire Genomics) located within the deleted region and standard techniques. TelVysion 12q (Abbot), located at subtelomeric region 12q, was used as a control probe. Parental metaphase FISH-analysis could not detect any deletion or any other rearrangement of the named region (data not shown).

\section{Discussion}

In this study we describe a de novo deletion at 12q12q13. The deleted region in our patient partially overlaps with previously described deletions in 5 independent cases 


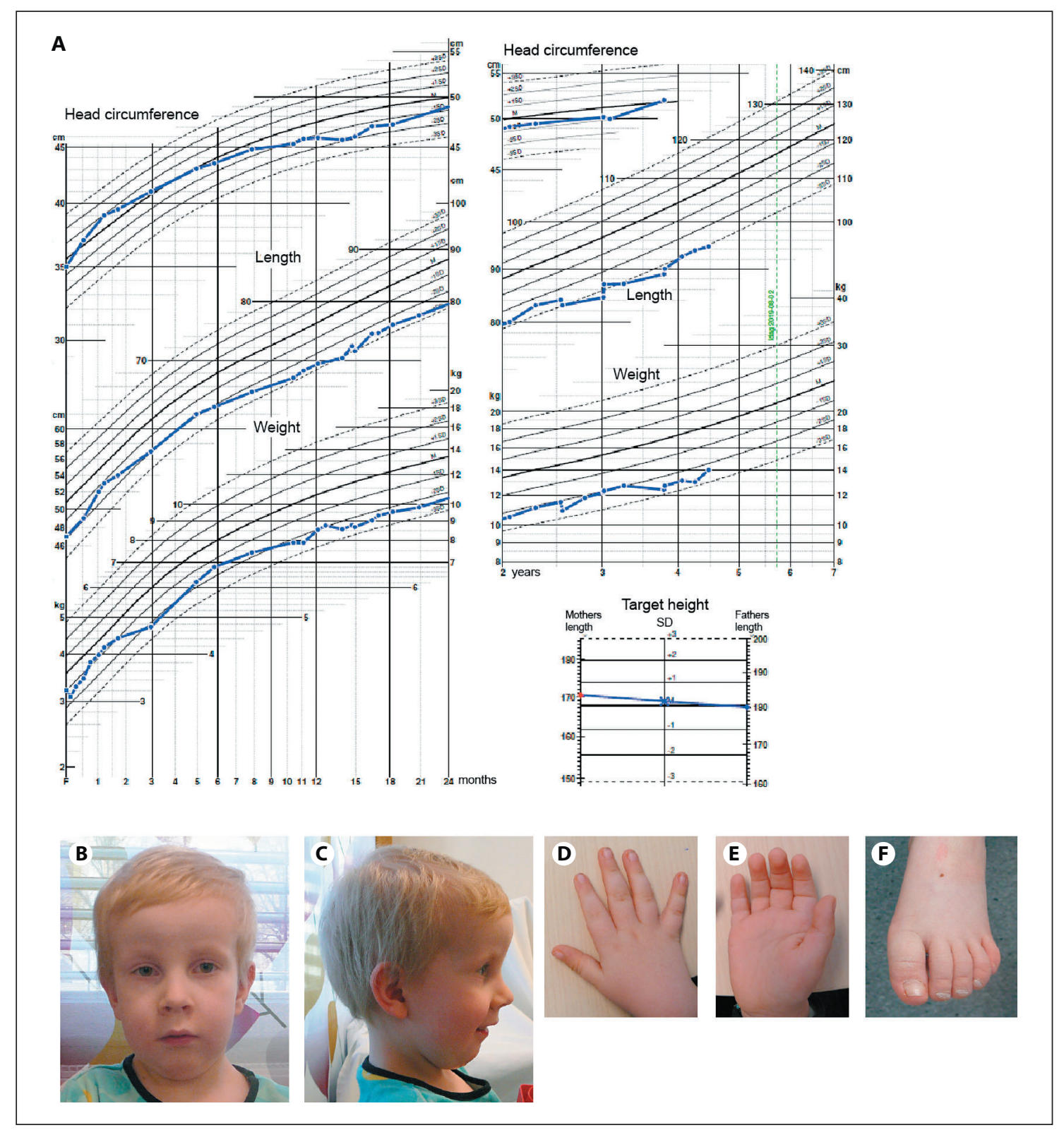

Fig. 1. A Growth curve representing the patient's growth parameters as well as a target height. B-F Pictures of the patient (facial and profile views, right hand and left foot) at age 6 years 7 months.

(Fig. 2) [Tonoki et al., 1998; Miyake et al., 2004; Failla et al., 2008; Carlsen et al., 2015; Weng et al., 2018]. Four cases, including our patient, presented with psychomotor developmental delay, short stature, decreased head circumferences, as well as large low-set ears, strabismus, broad nasal bridges and/or noses, long philtrums, downturned corners of the mouth, and widely spaced nipples (Table 1). The patient described by Carlsen et al. [2015] showed most of the above-mentioned clinical features, although he had small ears, a wide mouth and rather an increased head circumference. At a clinical investigation at age 10 years, his height and weight were within the normal range (10th centile).

Fifth-finger clinodactyly as well as small hands and/or feet were common features present in all of the 5 previously described cases, however, not present in our patient. Hypoplastic toenails as well as broad-based fingers were observed for our patient, but not reported for the 
Table 1. Clinical features of individuals with proximal 12q deletions

\begin{tabular}{|c|c|c|c|c|c|c|}
\hline & Present patient & Weng et al., 2018 & Carlsen et al., 2015 & Failla et al., 2008 & Miyake et al., $2004^{\mathrm{a}}$ & Tonoki et al., 1998 \\
\hline Position (size) & $\begin{array}{l}12 \mathrm{q} 12 \mathrm{q} 13.1 \\
(3.1 \mathrm{Mb})\end{array}$ & $\begin{array}{l}12 \mathrm{q} 12 \\
(3.18 \mathrm{Mb})\end{array}$ & $\begin{array}{l}12 \mathrm{q} 12 \\
\text { (1.13 MB, no } \\
\text { ARID2 deletion) }\end{array}$ & $\begin{array}{l}12 \mathrm{q} 12 \\
(4.5 \mathrm{Mb})\end{array}$ & $\begin{array}{l}12 \mathrm{q} 11 \mathrm{q} 13 \\
(\sim 10.5 \mathrm{Mb})\end{array}$ & $\begin{array}{l}12 \mathrm{q} 12 \mathrm{q} 13.12 \\
(\sim 6.3 \mathrm{Mb})\end{array}$ \\
\hline $\begin{array}{l}\text { Molecular coordinates } \\
\text { (hg19) }\end{array}$ & $\begin{array}{l}43,889,138- \\
47,011,108\end{array}$ & $\begin{array}{l}43,418,911- \\
46,601,627\end{array}$ & $\begin{array}{l}44,830,147- \\
45,964,945\end{array}$ & $\begin{array}{l}\sim 42,000,000- \\
\sim 47,000,000\end{array}$ & $\begin{array}{l}\sim 38,033,557- \\
\sim 48,566,447\end{array}$ & $\begin{array}{l}\sim 41,826,511- \\
\sim 48,084,277\end{array}$ \\
\hline Age/gender & 5 ys $3 \mathrm{~m} / \mathrm{M}$ & $3 \mathrm{~m} / \mathrm{F}$ & $10 \mathrm{ys} / \mathrm{M}$ & $10 \mathrm{ys} / \mathrm{M}$ & $20 \mathrm{~m} / \mathrm{unk}$ & $2 \mathrm{ys} / \mathrm{M}$ \\
\hline $\begin{array}{l}\text { Length/weight at birth, } \\
\text { SD }\end{array}$ & $-2 /-1$ & $-3 /-$ & $\begin{array}{l}-1 /-0.5 \\
\text { (10th/25-50th cen- } \\
\text { tile) }\end{array}$ & $\begin{array}{l}-1 /-1.5 \\
(10-25 \text { th } /<10 \text { th } \\
\text { centile })\end{array}$ & $-1-$ & $-3 /-3$ \\
\hline Head circumference, SD & -1 & -3 & +2 & -4 & NA & -2 \\
\hline Hypotonia & + & NA & + & + & $\begin{array}{l}\text { Hypertonia } \\
\text { (lower limbs) }\end{array}$ & + \\
\hline Speech delay & ++ (expressive $)$ & NR & + & + & NA & NA \\
\hline Intellectual disability & Border line & NR & Moderate & Moderate & NA & Severe \\
\hline Head & $\begin{array}{l}\text { Broad forehead } \\
\text { /scaphocephaly }\end{array}$ & Broad forehead & Broad forehead & $\begin{array}{l}\text { Microcephaly/ } \\
\text { brachycephaly }\end{array}$ & Broad forehead & Microcephaly \\
\hline Face & Long & NA & - & NA & NA & NA \\
\hline Large/low-set ears & $+/-$ & $+/+$ & small/+ & $+/+$ & $+/+$ & $+/+$ \\
\hline Hypertelorism & - & + & + & - & NA & + \\
\hline Strabismus & Slight & NA & + & + & + & + \\
\hline Palpebral fissures & Horizontal & Up-slanting & Down-slanting & Horizontal & Up-slanting & Down-slanting \\
\hline Flat/broad nasal bridge & Broad & Broad/depressed & Low/depressed & $-1-$ & NA & NA \\
\hline Abnormal nose shape & - & $\begin{array}{l}\text { High-inserted } \\
\text { columella }\end{array}$ & - & Broad & Short/broad & Short/broad \\
\hline Small hands/feet & $-1-$ & $+/+$ & $+/+$ & $-1+$ & $+/ \mathrm{NA}$ & $+/+$ \\
\hline Hypoplastic nails & $+($ toes $)$ & NA & NA & NA & NA & NA \\
\hline 5th finger clinodactyly & - & + & + & + & + & + \\
\hline Cardiac malformations & NA & $+(\mathrm{ASD})$ & + & NA & NA & - \\
\hline Feeding difficulties & + & + & + & + & NA & + \\
\hline Other & $\begin{array}{l}\text { Small pectus } \\
\text { excavatum }\end{array}$ & Small pectus excavatum & & & & \\
\hline
\end{tabular}

${ }^{\text {a }}$ Case 1 adapted from Gallego et al. [2000] as cited in Miyake et al. [2004]. ADHD, attention deficit hyperactivity disorder; ASD, atrial septal defect; m, months; NA, not assessed; NR, not relevant; , deducted position according to annotated deleted genes [Failla et al., 2008] or BAC-probes [Miyake et al., 2004]; unk, unknown; ys, years; +, present; -, absent. Bold print indicates information assessed from previously published patients' photos. 


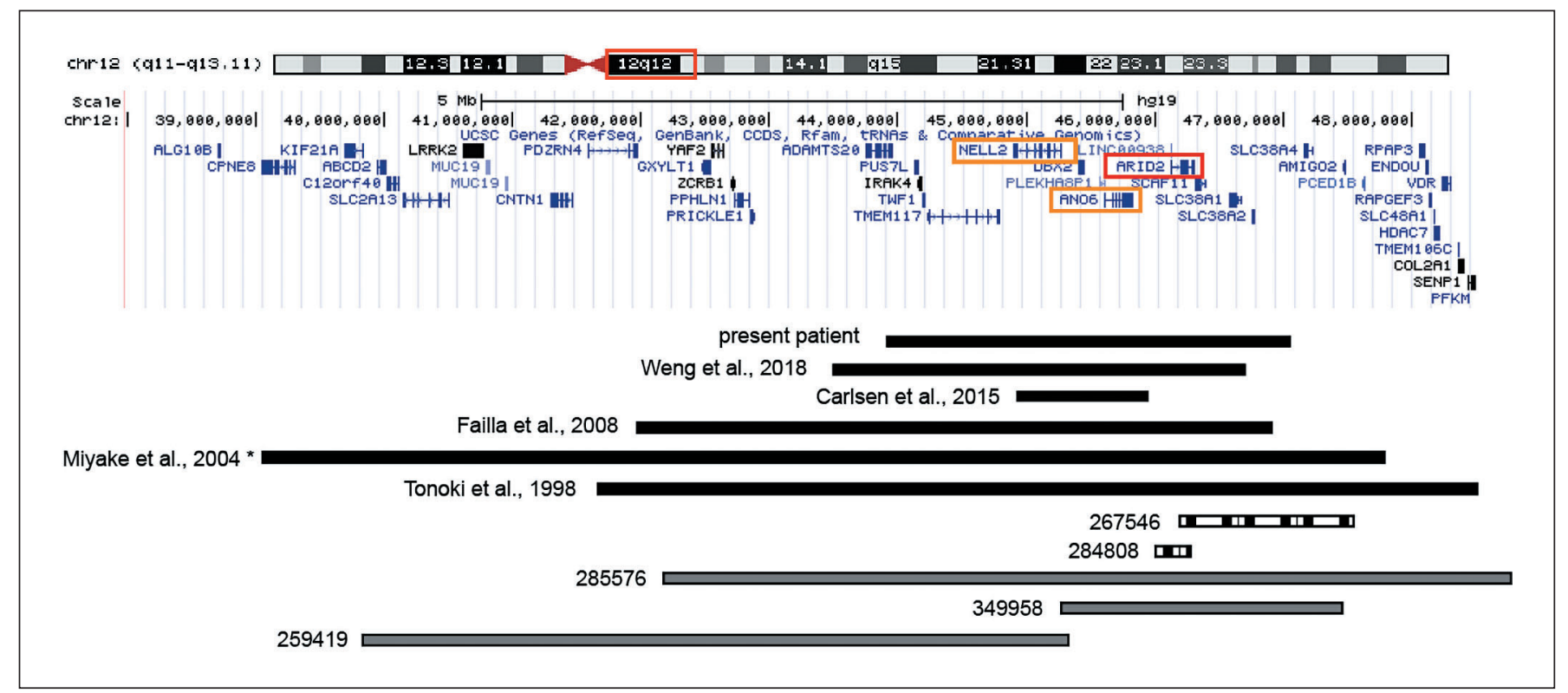

Fig. 2. Schematic illustration of gross proximal deletions at $12 \mathrm{q}$ described in the literature and present case. Black bars: published cases, lined bars: published DECIPHER cases, grey bars: unpublished DECIPHER cases.

other patients. Moreover, congenital atrial septal defect was present in one patient reported by Weng et al. [2018], and a dilated aortic root and a slightly thickened ventricular septum were detected in one patient at age 21 month reported by Carlsen et al. [2015].

The deletion spans 12 protein coding genes, of which 4, TWF1, TMEM117, NELL2, and ARID2, have haploinsufficiency scores below 25\% and are thereby likely to cause loss of function [Huang et al., 2010]. Moreover, 3 of the genes within the deleted region, ANO6, IRAK4, and $A R I D 2$, have previously been associated with known clinical conditions (OMIM morbid). ANO6 is associated with deficient platelet coagulation activity named Scott syndrome [Suzuki et al., 2010], which is a recessive condition. IRAK4 is involved in the functioning of immune system [Picard et al., 2003]. The symptoms in our patient do not match the phenotypes described for these disorders.

The minimal critical region and candidate genes for deletions at $12 \mathrm{q} 12$ were debated in at least 4 papers describing 4 patients with overlapping deletions (Fig. 2) [Miyake et al., 2004; Failla et al., 2008; Carlsen et al., 2015; Weng et al., 2018]. Miyake et al. [2004] suggested YAF2 and $A M I G O 2$ as possible candidates for the phenotypic condition in their 2 patients. The hypothesis of YAF2 to be associated with the growth retardation was also supported by Failla et al. [2008]. Additionally, they suggested PRICKLE1 to cause learning disability.

Proximal Deletion 12q and ARID2

Deficiency
Weng et al. [2018] as well as Carlsen et al. [2015] pointed out the importance of NELL2 in the physiology of the nervous system and possible association with growth retardation. A recent study showed the importance of NELL2 in appetite behaviour in rats and reduced food intake after the downregulation of NELL2 expression in the hypothalamus [Jeong et al., 2017]. Overall, only feeding difficulties were annotated in the patients with deletion $12 \mathrm{q}$ without any specific mention about essentially reduced appetite. Moreover, the patient reported by Carlsen et al. [2015], who carries a deletion involving NELL2, does not show severe growth retardation. Therefore, we presume that NELL2 is unlikely to have a major impact on the growth retardation described in this cohort.

ARID2 has not been considered as a strong candidate gene for the cohort of patients with $12 \mathrm{q}$ proximal gross deletions before. However, ARID2 is the only overlapping gene in the 4 previously reported cases to be associated with the disorder [Bramswig et al., 2017; Van Paemel et al., 2017; Gazdagh et al., 2019]. ARID2 was not deleted in the patient described by Carlsen et al. [2015] (Fig. 2). Interestingly, that patient neither presented with moderate nor severe growth retardation (Table 1); his growth parameters were even normalised by the age of 10 , and he has an increased head circumference. 
Table 2. Clinical features of individuals with ARID2 variants

\begin{tabular}{|c|c|c|c|c|c|c|c|c|}
\hline & \multirow{2}{*}{$\begin{array}{l}\text { Present } \\
\text { patient }\end{array}$} & \multirow[t]{2}{*}{ Decipher $267546^{a}$} & \multirow[t]{2}{*}{ Decipher $284808^{a}$} & \multirow{2}{*}{$\begin{array}{l}\text { Van Paemel et al., } \\
2017\end{array}$} & \multicolumn{4}{|l|}{ Shang et al., 2015} \\
\hline & & & & & 1 & 2 & 3 & 4 \\
\hline Genetic abnormality & $\begin{array}{l}\text { 3.1-Mb } \\
\text { deletion } \\
(A R I D 2 \text {, entire } \\
\text { gene) }\end{array}$ & $\begin{array}{l}\text { 1.39-Mb deletion } \\
\text { (ARID2, } \\
\text { exons } 4-21)\end{array}$ & $\begin{array}{l}\text { 246-kb deletion } \\
\text { (ARID2, } \\
\text { exons } 1-16)\end{array}$ & $\begin{array}{l}\text { 105-kb deletion } \\
\text { (ARID2, } \\
\text { exons } 3-5)\end{array}$ & $\begin{array}{l}\text { c.2536delG; } \\
\text { p.Val846Leufs*3 }\end{array}$ & $\begin{array}{l}\text { c. } 1028 \mathrm{~T}>\mathrm{A} \\
\text { p.Leu343* }\end{array}$ & $\begin{array}{l}\text { c. } 4441 \text { delC; } \\
\text { p.His1481Ilefs*4 }\end{array}$ & $\begin{array}{l}\text { c. } 4318 \mathrm{C}>\mathrm{T} \\
\text { p.Q1440* }\end{array}$ \\
\hline Age/gender & 5 ys $3 \mathrm{~m} / \mathrm{M}$ & 23 ys/F & $4.5 \mathrm{ys} / \mathrm{M}$ & 3 ys $11 \mathrm{~m} / \mathrm{F}$ & $15 \mathrm{ys} / \mathrm{F}$ & $8 \mathrm{ys} / \mathrm{F}$ & $6 \mathrm{ys} / \mathrm{F}$ & $8 \mathrm{ys} / \mathrm{F}$ \\
\hline Height/weight, SD & $-3 /-2.2$ & $-1 / \mathrm{NA}$ & $-3.6 / \mathrm{NA}$ & $-3.3 /-4$ & $-2 / \mathrm{NA}$ & $-1 /-1$ & $-1 /-2$ & $-1 /-2$ \\
\hline $\begin{array}{l}\text { Length/weight at birth, } \\
\text { SD }\end{array}$ & $-2 /-1$ & $\mathrm{NA} /-2$ & $\mathrm{NA} /-4$ & $-2.2 /-1.4$ & $\mathrm{NA} /-2$ & $\mathrm{NA} /-1$ & $\mathrm{NA} /-1$ & $\mathrm{NA} /-1$ \\
\hline Head circumference, SD & -1 & -0.5 & -0.05 & -2.0 & NA & NA & NA & NA \\
\hline Hypotonia & + & + & NA & + & + & NA & - & + \\
\hline Behaviour & $\begin{array}{l}\text { Autism } \\
\text { (atypical) }\end{array}$ & Anxiety & Sleep disturbance & $\begin{array}{l}\text { ADHD/noise } \\
\text { sensitivity }\end{array}$ & ADHD/anxiety & $\begin{array}{l}\text { ADHD/ } \\
\text { aggressive }\end{array}$ & $\begin{array}{l}\text { Water affinity/ } \\
\text { noise sensitivity }\end{array}$ & ADHD/tics \\
\hline $\begin{array}{l}\text { Delayed motor } \\
\text { development }\end{array}$ & $\begin{array}{l}\text { Mild (oral), } \\
\text { dyspraxia }\end{array}$ & NA & + & + & + & + & + & + \\
\hline Cognitive delay & + & NA & NA & + & + & + & + & + \\
\hline Speech delay & ++ (expressive) & NA & + & + & + & NA & + & NA \\
\hline Intellectual disability & Border line & $\begin{array}{l}\text { Moderate learning } \\
\text { difficulties }\end{array}$ & NA & NA & + & NA & NA & NA \\
\hline Head & $\begin{array}{l}\text { Broad/high } \\
\text { forehead/ } \\
\text { scafocephaly }\end{array}$ & Broad forehead & Broad forehead & High forehead & Frontal bossing & Frontal bossing & Frontal bossing & Frontal bossing \\
\hline Face & Long & Long & - & Coarse & NA & Coarse & Coarse & NA \\
\hline Large/low-set ears & $+1-$ & $\mathrm{NA} /+$ & $-1+$ & $\mathrm{NA} /-$ & + & $\mathrm{NA} /+$ & $+/+$ & + \\
\hline Hypertelorism & - & - & - & - & NA & NA & + & NA \\
\hline Strabismus & Slight & NA & NA & + & NA & NA & NA & NA \\
\hline Palpebral fissures & Horizontal & Down-slanting & Horizontal & Down-slanting & Down-slanting & Down-slanting & Down-slanting & Down-slanting \\
\hline Flat/broad nasal bridge & Broad & - & - & - & NA & - & $-1+$ & NA \\
\hline Abnormal nose shape & - & Long & - & Broad tip & NA & NA & NA & NA \\
\hline Anteverted nares & - & - & - & - & NA & - & - & NA \\
\hline Long philtrum & + & + & + & Prominent & NA & Flat & Flat & NA \\
\hline Mouth size/chin & Micrognathia & Micrognathia & Micrognathia & Retrognathia & Micrognathia & Retrognathia & Micrognathia & Micrognathia \\
\hline Mouth shape & Normal & Thin upper lip & Thin upper lip & Normal & NA & Wide & Thin upper lip & NA \\
\hline Cleft/high-arched palate & + (high) & + & NA & - & NA & + & NA & NA \\
\hline Widely set nipples & + & NA & NA & NA & NA & NA & NA & NA \\
\hline Small hands/feet & $-1-$ & Arachnodactyly & NA & NA & NA & NA & NA & NA \\
\hline Hypoplastic nails & + (toes) & $+($ toes $)$ & + (toes) & + & NA & NA & NA & NA \\
\hline 5th finger clinodactyly & - & NA & NA & NA & NA & NA & NA & NA \\
\hline Cardiac malformations & NA & NA & NA & NA & NA & $+(\mathrm{ASD})$ & NA & NA \\
\hline Feeding difficulties & + & NA & + , laryngomalacia & $\begin{array}{l}+ \text {, oromotor } \\
\text { dysfunction }\end{array}$ & + & + & + & + \\
\hline Other & $\begin{array}{l}\text { Small pectus } \\
\text { excavatum }\end{array}$ & & & $\begin{array}{l}\text { Clubfoot unilateral, } \\
\text { bilateral hip } \\
\text { dysplasia }\end{array}$ & & & & \\
\hline
\end{tabular}

ARID2 encodes an AT-rich interactive domain (ARID)containing DNA-binding protein required for the stabilisation of SWI/SNF chromatin remodelling complex SWI/SNF-B (PBAF), which regulates embryonic cell patterning and cell cycle control [Xu et al., 2012; You et al., 2013; Cabot et al., 2017]. It has been associated with short stature, intellectual disability, and specific dysmorphic features [Shang et al., 2015; Gazdagh et al., 2019]. However, only a few patients with ARID2 deficiency have been described. Common clinical symptoms for these cases are short stature and global developmental delay with cognitive impairment [Shang et al., 2015; Bramswig et al., 2017; Van Paemel et al., 2017; Gazdagh et al., 2019]. They also share dysmorphic features such as a coarse face with prominent forehead, broad nose, and down-slanting palpebral fissures (Table 2). Micrognathia, abnormal philtrum and posteriorly rotated low-set ears were present in most of the patients. Moreover, a majority of the patients present with hypotonia, feeding difficulties, and various behavioural abnormalities. 
Table 2 (continued)

\begin{tabular}{|c|c|c|c|c|c|c|c|}
\hline & \multicolumn{2}{|l|}{ Bramswig et al., 2017} & \multirow[t]{2}{*}{ DDD $267395^{a}$} & \multirow[t]{2}{*}{ DDD $275526^{a}$} & \multirow[t]{2}{*}{ DDD $263830^{\mathrm{a}}$} & \multirow[t]{2}{*}{ DDD $265539^{a}$} & \multirow[t]{2}{*}{ DDD $272339^{a}$} \\
\hline & 1 & 2 & & & & & \\
\hline Genetic abnormality & $\begin{array}{l}\text { c.3411_3412delAG; } \\
\text { p.Gly1139Serfs*20 }\end{array}$ & $\begin{array}{l}\text { c.156delC; } \\
\text { p.Arg53Glufs*5 }\end{array}$ & $\begin{array}{l}\text { c.1158dup; } \\
\text { p.(Asn387*) }\end{array}$ & $\begin{array}{l}\text { c. } 399 \mathrm{C}>\mathrm{G} ; \\
\text { p. }(\text { Tyr133*) }\end{array}$ & $\begin{array}{l}\text { c. } 4444 \mathrm{C}>\mathrm{T} ; \\
\text { p. }\left(\mathrm{G} \ln 1482^{*}\right)\end{array}$ & $\begin{array}{l}\text { c.4687_4690dup; } \\
\text { p.(Thr1564Lysfs*5) }\end{array}$ & $\begin{array}{l}\text { c.2645_2646insCT; } \\
\text { p.(Val883Leufs*10) }\end{array}$ \\
\hline Age/gender & $7 \mathrm{ys} / \mathrm{M}$ & $4 \mathrm{ys} / \mathrm{M}$ & $2 \mathrm{ys} / \mathrm{M}$ & 6 ys $10 \mathrm{~m} / \mathrm{M}$ & 8 ys $3 \mathrm{~m} / \mathrm{M}$ & 3 ys $11 \mathrm{~m} / \mathrm{M}$ & $18 \mathrm{ys} / \mathrm{M}$ \\
\hline Height/weight, SD & $-2.14 / \mathrm{NA}$ & $-2.2 / \mathrm{NA}$ & $-2 /-1$ & $-2 /-1$ & $-1.37 /+0.94$ & $-2 / \mathrm{NA}$ & $+0.74 /+1.5$ \\
\hline $\begin{array}{l}\text { Length/weight at birth, } \\
\text { SD }\end{array}$ & $-1.6 /-1.6$ & $-0.7 /+2.1$ & $\mathrm{NA} /-0.53$ & $\mathrm{NA} /-1$ & $\mathrm{NA} /+0.16$ & $\mathrm{NA} /-2.2$ & $\mathrm{NA} /-1.16$ \\
\hline $\begin{array}{l}\text { Head circumference, } \\
\text { SD }\end{array}$ & +0.68 & -0.4 & +0.3 & +2 & +0.65 & NA & +0.04 \\
\hline Hypotonia & + & + & + & NA & NA & NA & NA \\
\hline Behaviour & Inability to sit alone & + & $\begin{array}{l}\text { "Routine driven"/ } \\
\text { rigid/anxiety }\end{array}$ & $\begin{array}{l}\text { "Routine driven"/ } \\
\text { noise sensitivity }\end{array}$ & / Rigid/anxiety & NA & Quiet \\
\hline $\begin{array}{l}\text { Delayed motor } \\
\text { development }\end{array}$ & + & + & + & + & + & + & - \\
\hline Cognitive delay & + & + & + & + & + & + & + \\
\hline Speech delay & + & + & + & ++ & ++ & + & ++ \\
\hline Intellectual disability & Severe & ++ & Moderate & Moderate & + & + & + \\
\hline Head & High forehead & $\begin{array}{l}\text { Large forehead/ } \\
\text { frontal bossing }\end{array}$ & Plagiocephaly & Broad forehead & NA & High/broad forehead & NA \\
\hline Face & Coarse & Coarse/triangular & Non-coarse & Coarse & NA & Coarse & Coarse \\
\hline Large/low-set ears & $-1+$ & - & Protruding & $\mathrm{NA} /+$ & NA/NA & Posteriorly rotated & NA \\
\hline Hypertelorism & + & - & - & NA & NA & NA & NA \\
\hline Strabismus & - & - & NA & + & NA & NA & NA \\
\hline Palpebral fissures & Narrow & $\begin{array}{l}\text { Horizontal, } \\
\text { narrow }\end{array}$ & NA & Down-slanting & NA & NA & NA \\
\hline Flat/broad nasal bridge & $+/-$ & + & NA & NA & NA & $+/+$ & NA \\
\hline Abnormal nose shape & Broad, short & Broad, short & NA & NA & NA & Broad tip & Broad tip \\
\hline Anteverted nares & + & + & NA & NA & NA & + & NA \\
\hline Long philtrum & $+/$ prominent & Prominent & NA & NA & NA & Broad & NA \\
\hline Mouth size/chin & Large/- & Large/- & NA & NA & Micrognathia & NA & Micrognathia \\
\hline Mouth shape & $\begin{array}{l}\text { Thin upper, full } \\
\text { lower lips }\end{array}$ & $\begin{array}{l}\text { Thin upper lip, } \\
\text { thick lower lip }\end{array}$ & $\begin{array}{l}\text { Thin upper lip } \\
\text { vermilion; downturned } \\
\text { corners }\end{array}$ & NA & NA & NA & $\begin{array}{l}\text { Thick lower lip } \\
\text { vermilion }\end{array}$ \\
\hline $\begin{array}{l}\text { Cleft/high-arched } \\
\text { palate }\end{array}$ & - & - & NA & NA & NA & NA & NA \\
\hline Widely set nipples & NA & NA & NA & NA & NA & NA & NA \\
\hline Small hands/feet & $+/+$ & NA & NA & NA & Short toes & NA & NA \\
\hline Hypoplastic nails & + & + & NA & $+($ toes $)$ & + (fingers) & small nails $2^{\text {nd }}$ toes & NA \\
\hline $5^{\text {th }}$ finger clinodactyly & NA & + & NA & NA & NA & + & NA \\
\hline Cardiac malformations & NA & NA & NA & NA & NA & NA & NA \\
\hline Feeding difficulties & + & - & NA & NA & NA & + , poor swallow & NA \\
\hline Other & $\begin{array}{l}\text { Dandy-Walker } \\
\text { anomaly, abnormal } \\
\text { corpus callosum }\end{array}$ & & $\begin{array}{l}\text { Constipations, frequent } \\
\text { infections, myotonic } \\
\text { dystrophy }\end{array}$ & $\begin{array}{l}\text { Dysmorphic } \\
\text { corpus callosum, } \\
\text { delayed } \\
\text { myelination }\end{array}$ & $\begin{array}{l}\text { Webbed neck, } \\
\text { Wormian bones }\end{array}$ & Myopia, hypermetropia & $\begin{array}{l}\text { Low anterior hairline, } \\
\text { thick eyebrows, pes } \\
\text { cavus, joint } \\
\text { hypermobility, } \\
\text { myopia }\end{array}$ \\
\hline
\end{tabular}

a Adapted from Gazdagh et al., 2019. Deleted exons were deducted according to array breakpoints using USCG Genome Browser (GRCh37/h19). ARID2 SNVs correspond to NM_152641.3 transcript. ADHD, attention deficit hyperactivity disorder; ASD, atrial septal defect; DDD, deciphering developmental disorders; m, months; NA, not assessed; ys, years. +, present; -, absent.

Both cohorts with ARID2 SNVs and with whole gene deletions (del12q) share distinct facial dysmorphic features such as prominent forehead, low-set ears, broad nose, long/prominent philtrum, and small mouth. Small hands and feet are much more common in patients with del12q whereas down-slanting palpebral fissures were only present in 2 cases. Coarse face was not typical for the del12q group, whereas widely spaced nipples were not annotated for the ARID2 SNVs group. Hypotonia and feeding difficulties are features annotated for most of the pa- 


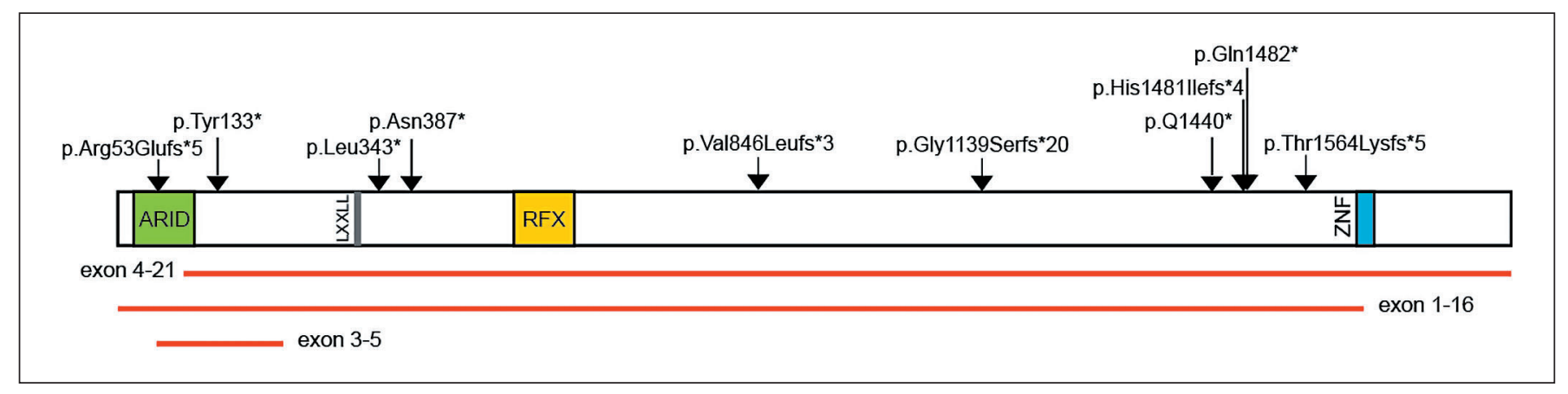

Fig. 3. ARID2 protein structure (adapted from UniProtKB Q68CP9) and distribution of pathogenic variants across the protein. Functional regions: ARID: AT-rich DNA interaction domain, LXXLL: nuclear receptor recognition motif, RFX: winged-helix-DNA-binding domain, ZNF: C2H2 zinc finger region. Position of SNVs reported by Shang et al. [2015], Bramswig et al. [2017], and Gazdagh et al. [2019] are marked with arrows. Intragenic deletions reported by Van Paemel et al. [2017] and Gazdagh et al. [2019] are indicated with red lines and deleted exons are annotated.

tients in both groups. Developmental delay was observed in all patients.

Severe growth retardation $(<-3 S D)$ was not present neither in the ARID2 SNV s cohort nor in the patient from Carlsen et al. [2015] who had an intact ARID2. Furthermore, one patient with an intragenic deletion in ARID2 (DECIPHER 267546) did not show severe growth retardation. Instead these patients were growing in the range of -1 SD to -2.2 SD.

ARID2 deficiency is also correlated with Coffin-Siris syndrome (CSS; MIM 617808) pointing out global developmental delay, short stature, and coarse facial features [Bramswig et al., 2017; Van Paemel et al., 2017]. CSS is caused by pathogenic variants in different components of the SWI/SNF-BAF complex, including ARID1A and ARID1B [Santen et al., 2013]. Since ARID2 is a component of the PBAF subunit of the SWI/SNF complex, coexpression of ARID2 with ARID1A and other components of the BAF complex suggests a phenotypic overlap such as intellectual disability for CSS and ARID2 deficiency [Lessard et al., 2007; Bramswig et al., 2017; Van Paemel et al., 2017]. However, typical CSS features like hypertrichosis, sparse scalp hair, long eyelashes, and bushy eyebrows are present neither in the ARID2 SNVs nor in the del12q group.

ARID2-containing SWI/SNF-PBAF protein complex regulates tissue-specific gene expression. Depletion of ARID2 affects expression of the anabolic growth factor $B M P 4$ and the growth factor receptor FGFR2 being critical for osteoblast differentiation, particularly pre-osteoblast commitment [Xu et al., 2012]. The same study dem- onstrated a negative effect of ARID2 depletion on the mineralisation phenotype in maturing osteoblasts. These facts might explain the growth abnormality and craniofacial features in patients carrying heterozygous variants in ARID2. Complete ARID2 knockout mice display severe cardiac defects with reduced proliferation of cardiomyocytes and embryonic lethality [He et al., 2014]. Implication of ARID2 in the cardiac system development could explain observed heart defects in some patients (Tables 1 , 2 ). There are no described cases with homozygous depletion of ARID2 in humans.

Remarkably, all described SNVs in ARID2 (frameshift and nonsense) have a deleterious effect. The variant c.3411_3412delAG (p.Gly1139Serfs*20) was even hypothesised to be attended by nonsense-mediated RNA decay (NMD) leading to haploinsufficiency of ARID2 [Bramswig et al., 2017]. A similar mechanism for a deleterious effect may be speculated for other SNVs. However, detailed analysis of the NMD phenomenon for any ARID2 SNV was not performed.

In fact, the different grade of growth retardation in the 2 groups of patients may indicate different effects of SNVs or intragenic deletions and gross deletions on ARID2 functional implication in the pathogenesis. Analysis of the location of SNVs and deletions in correlation with ARID2 structure may shed new light to the prediction of possible functional effects of these genetic variants. ARID2 is an 1835 aa protein containing 4 distinguished functional regions (Fig. 3). Three highly conservative DNA-binding domains (ARID, RFX, and ZNF) together with a nuclear receptor recognition motif (LXXLL) medi- 
ate transcriptional activation of selected genes [Emery et al., 1996; Savkur and Burris, 2004; Patsialou et al., 2005].

Most SNVs located between the LXXLL and ZNF regions are predicted to cause a loss of the ZNF domain. The exceptions are the p.Arg53Glufs* 5 variant located in the ARID domain and p.Tyr133* located just after the ARID domain. These patients together with the patient carrying the p.Gly1139Serfs*20 variant, with a hypothesised NMD effect, show growth retardation just below -2 $\mathrm{SD}$, which is more severe compared to patients with other ARID2 SNVs [Bramswig et al., 2017]. Most published cases with intragenic deletions within ARID2, span either only the ARID domain (exons 3-5 deleted) or ARID together with LXXLL and RFX regions (exons 1-16 deleted) [Van Paemel et al., 2017; Gazdagh et al., 2019]. However, DECIPHER patient 267546 does not have a severe growth retardation, though all 4 functional regions are deleted (exons 4-21 deleted). Very little is known about the growth parameters for this patient during the first and second decades of her life. Also, we do not know whether she received a hormonal therapy in a view of a short stature. Growth parameters presented in Table 2 correspond to 23 years, whereas all other patients have been examined on younger age. We admit that growth is a complex and dynamic trait which is influenced by both genetic and environmental factors. Thus, for a stronger comparative and correlative analysis, growth and developmental parameters would have to be compared for every patient at the same age. Some of the included patients were shown to carry other genetic variants, both CNVs and SNVs, unrelated to the $12 \mathrm{q} 11 \mathrm{q} 13$ region. These variants were interpreted as likely benign based on their functional score and/or mode of inheritance [Shang et al., 2015; Bramswig et al., 2017; Gazdagh et al., 2019]. However, we cannot completely exclude possible functional impact of these or other genetic variants on growth or development.

Taken together our observations corroborate that gross deletions at $12 \mathrm{q} 11 \mathrm{q} 13$ represent a distinct clinical subgroup of genetic abnormalities. Described deletions span different genes, and some clinical variations are the same or differ in between patients. Growth retardation $(<-2 \mathrm{SD})$ is present in 5 out of 6 patients suggesting a critical region for this clinical feature with ARID2 as a possible candidate gene. The present study is the first attempt to draw a connection between gross deletions at $12 \mathrm{q} 12 \mathrm{q} 13$ and ARID2 deficiency. Comparative analysis of phenotypes for both ARID2 SNVs and del12q groups indicates craniofacial, skeletal and central nervous system abnormalities with high similarity between the 2 cohorts. However, a growth retardation $<-2$ SD seems to be pres- ent mainly in patients with gross deletions, partially or completely spanning the ARID2 gene. Further characterisation of the effect of above-described genetic variants on ARID2 expression and protein structure would bring us a better understanding of pathogenesis in patients carrying proximal 12q11q13 deletions.

\section{Acknowledgement}

We are very grateful to the family participating in the study.

\section{Statement of Ethics}

This study was approved by the ethics committee. Written parental consent was obtained.

\section{Disclosure Statement}

The authors have no conflicts of interest to declare.

\section{Funding Sources}

M.S., A.C.T., and C.S.Z. were supported by Arbetslivsfonden (ALF) funding.

\section{Author Contributions}

M.S. compiled the study. C.S.Z. and N.P. examined and described the patient. M.S. and A.C.T. analysed genetic testing results. M.S., A.C.T., and C.S.Z. wrote the manuscript.

Bramswig NC, Caluseriu O, Lüdecke HJ, Bolduc
FV, Noel NC, et al: Heterozygosity for ARID2
loss-of-function mutations in individuals
with a Coffin-Siris syndrome-like phenotype.
Hum Genet 136:297-305 (2017).
Cabot B, Tseng YC, Crodian JS, Cabot R: Differ-
ential expression of key subunits of SWI/SNF
chromatin remodeling complexes in porcine
embryos derived in vitro or in vivo. Mol Re-
prod Dev 84:1238-1249 (2017).
Carlsen EØ, Frengen E, Fannemel M, Misceo D:
Haploinsufficiency of ANO6, NELL2 and
DBX2 in a boy with intellectual disability and
growth delay. Am J Med Genet A 167A:1890-
1896 (2015).
Emery P, Durand B, Mach B, Reith W: RFX pro-
teins, a novel family of DNA binding proteins
conserved in the eukaryotic kingdom. Nucle-
ic Acids Res 24:803-807 (1996).


Failla P, Romano C, Reitano S, Di Benedetto D, Grillo L, et al: 12q12 deletion: a new patient contributing to genotype-phenotype correlation. Am J Med Genet A 146A:1354-1357 (2008).

Gazdagh G, Blyth M, Scurr I, Turnpenny PD, Mehta SG, et al: Extending the clinical and genetic spectrum of ARID2 related intellectual disability. A case series of 7 patients. Eur J Med Genet 62:27-34 (2019).

- He L, Tian X, Zhang H, Hu T, Huang X, et al: BAF200 is required for heart morphogenesis and coronary artery development. PLoS One 9:e109493 (2014)

Huang N, Lee I, Marcotte EM, Hurles ME: Characterising and predicting haploinsufficiency in the human genome. PLoS Genet 6:e1001154 (2010).

Jeong JK, Kim JG, Kim HR, Lee TH, Park JW, Lee BJ: A role of central NELL2 in the regulation of feeding behavior in rats. Mol Cells 40:186194 (2017).

Lessard J, Wu JI, Ranish JA, Wan M, Winslow MM, et al: An essential switch in subunit composition of a chromatin remodeling complex during neural development. Neuron 55:201215 (2007).
Lynch SA, Foulds N, Thuresson AC, Collins AL, Annerén G, et al: The 12q14 microdeletion syndrome: six new cases confirming the role of HMGA2 in growth. Eur J Hum Genet 19: 534-539 (2011).

Miyake N, Tonoki H, Gallego M, Harada N, Shimokawa O, et al: Phenotype-genotype correlation in two patients with $12 \mathrm{q}$ proximal deletion. J Hum Genet 49:282-284 (2004).

Patsialou A, Wilsker D, Moran E: DNA-binding properties of ARID family proteins. Nucleic Acids Res 33:66-80 (2005).

- Picard C, Puel A, Bonnet M, Ku CL, Bustamante $\mathrm{J}$, et al: Pyogenic bacterial infections in humans with IRAK-4 deficiency. Science 299: 2076-2079 (2003).

- Santen GW, Aten E, Vulto-Van Silfhout AT, Pottinger C, Van Bon BW, et al: Coffin-Siris syndrome and the BAF complex: genotype-phenotype study in 63 patients. Hum Mutat 34: 1519-1528 (2013).

Savkur RS, Burris TP: The coactivator LXXLL nuclear receptor recognition motif. J Pept Res 63:207-212 (2004).
Shang L, Cho MT, Retterer K, Folk L, Humberson $\mathrm{J}$, et al: Mutations in ARID2 are associated with intellectual disabilities. Neurogenetics 16:307-314 (2015)

- Suzuki J, Umeda M, Sims PJ, Nagata S: Calciumdependent phospholipid scrambling by TMEM16F. Nature 468:834-838 (2010).

Tonoki H, Saitoh S, Kobayashi K: Patient with $\operatorname{del}(12)(q 12 q 13.12)$ manifesting abnormalities compatible with Noonan syndrome. Am J Med Genet 75:416-418 (1998).

Van Paemel R, De Bruyne P, Van Der Straaten S, D'hondt M, Frankel U, et al: Confirmation of an ARID2 defect in SWI/SNF-related intellectual disability. Am J Med Genet A 173:31043108 (2017).

-Weng Y, Luo X, Hou L: Deletion at 12q12 increases the risk of developmental delay and intellectual disability. Ann Hum Genet 82:482487 (2018).

Xu F, Flowers S, Moran E: Essential role of ARID2 protein-containing SWI/SNF complex in tissue-specific gene expression. J Biol Chem 287: 5033-5041 (2012).

- You JS, De Carvalho DD, Dai C, Liu M, Pandiyan $\mathrm{K}$, et al: SNF5 is an essential executor of epigenetic regulation during differentiation. PLoS Genet 9:e1003459 (2013). 